\title{
Intensive Care Medicine in 2050: global perspectives
}

\author{
Yaseen M. Arabi ${ }^{1,2^{*}}$, Marcus J. Schultz ${ }^{3,4}$ and Jorge I. F. Salluh ${ }^{5,6}$
}

C 2016 Springer-Verlag Berlin Heidelberg and ESICM

\section{The changing world}

With a projected increase in the world population from 7.2 billion currently to 9.6 billion by 2050, the population of the 49 least developed countries is expected to double from 900 million in 2013 to 1.8 billion [1]. As such, the number of patients who may need critical care will increase substantially, with a majority of these in resource-restricted settings. Coupled with an ageing global population, the complexity of the challenge will be enormous. Infectious diseases and trauma will continue to be main reasons for intensive care unit (ICU) admission, but natural as well as man-made disasters will occasionally place additional strain on critical care services, with the largest strain again in resourcerestricted settings. Healthcare expenditures vary considerably from approximately US $\$ 9400$ annually per capita in the USA to less than US $\$ 20$ per capita in many African countries [2], and with limited financial resources, the strategic priority is given to public health programs and not to more expensive services such as critical care. Around the globe, what constitutes an ICU will continue to vary in terms of structure, processes and staffing, all of which often remain inadequate in resource-restricted settings [3]. However, the basic premise will remain the same: the outcome of critically ill patients will substantially be improved by the provision of essential critical care [4], and many aspects can and should be provided even if resources are low [5]. Moving towards better critical care globally will require a multifaceted approach to address not only patient and organizational challenges, but also those related to staffing, cultural and technological aspects, as well as quality and safety. Addressing these

\footnotetext{
*Correspondence: Arabi@ngha.med.sa

1 Department of Intensive Care, King Saud bin Abdulaziz University for Health Sciences (KSAU-HS), PO Box 22490, Riyadh 11426, Saudi Arabia Full author information is available at the end of the article
}

complex challenges will certainly require involvement not only at the local ICU level but also at the level of hospitals, national societies and governments. In Table 1, we present examples of where and how critical care could and should improve, with a focus on resource-restricted settings. Some of these cases are highlighted in the following text.

\section{Patient level challenges and solutions}

Patient-level challenges to critical care in 2050, including increasing patient numbers and limited or no access to critical care, must be addressed by means other than merely increasing the number of beds per capita. Acute care services vary substantially across regions, and the variation is only partially attributable to differences in the gross domestic product [6]. Focus needs to be on early pre-ICU intervention and on better triage in order to reduce morbidity and the need for ICU admission. In addition, improving resource utilization and reducing waste will be equally essential. Finally, a strategy of appropriate end-of-life care that maintains patient integrity but avoids futile interventions needs to be introduced, especially in settings where clear standards do not yet exist.

\section{ICU-level challenges and solutions}

Lack of proper basic infrastructures, including equipment and supplies, and substandard infection control will continue to challenge critical care by 2050 . Prioritization will vary depending on the setting. In some areas, availing of intravenous fluids and uninterrupted oxygen and electricity may provide the greatest return. For mechanically ventilated patients, simply improving limited or unsafe endotracheal suctioning will prove to be life saving. There is a clear need for setting-specific standards for what is minimally needed within an ICU to be adopted by hospitals and governments.

\section{实




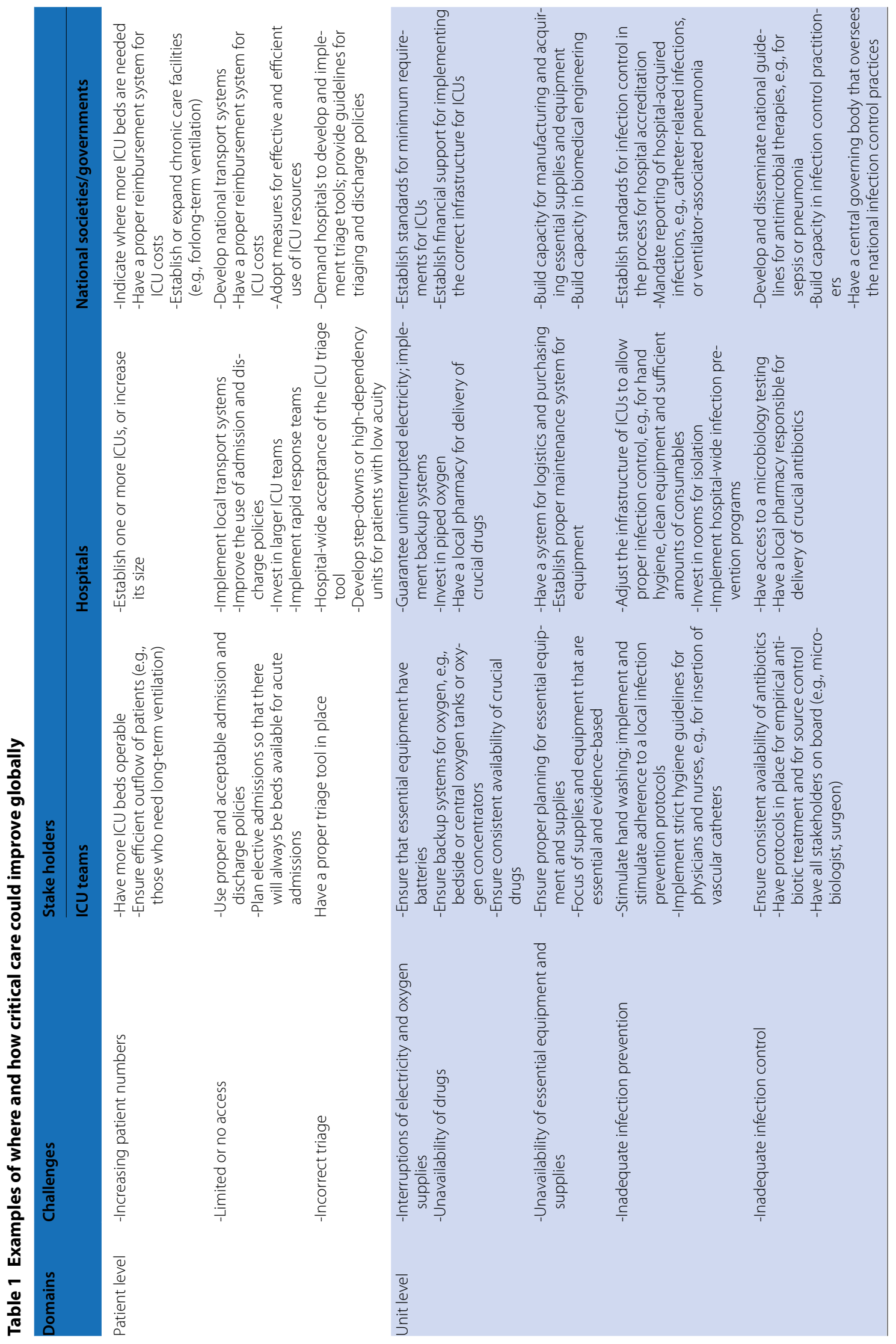




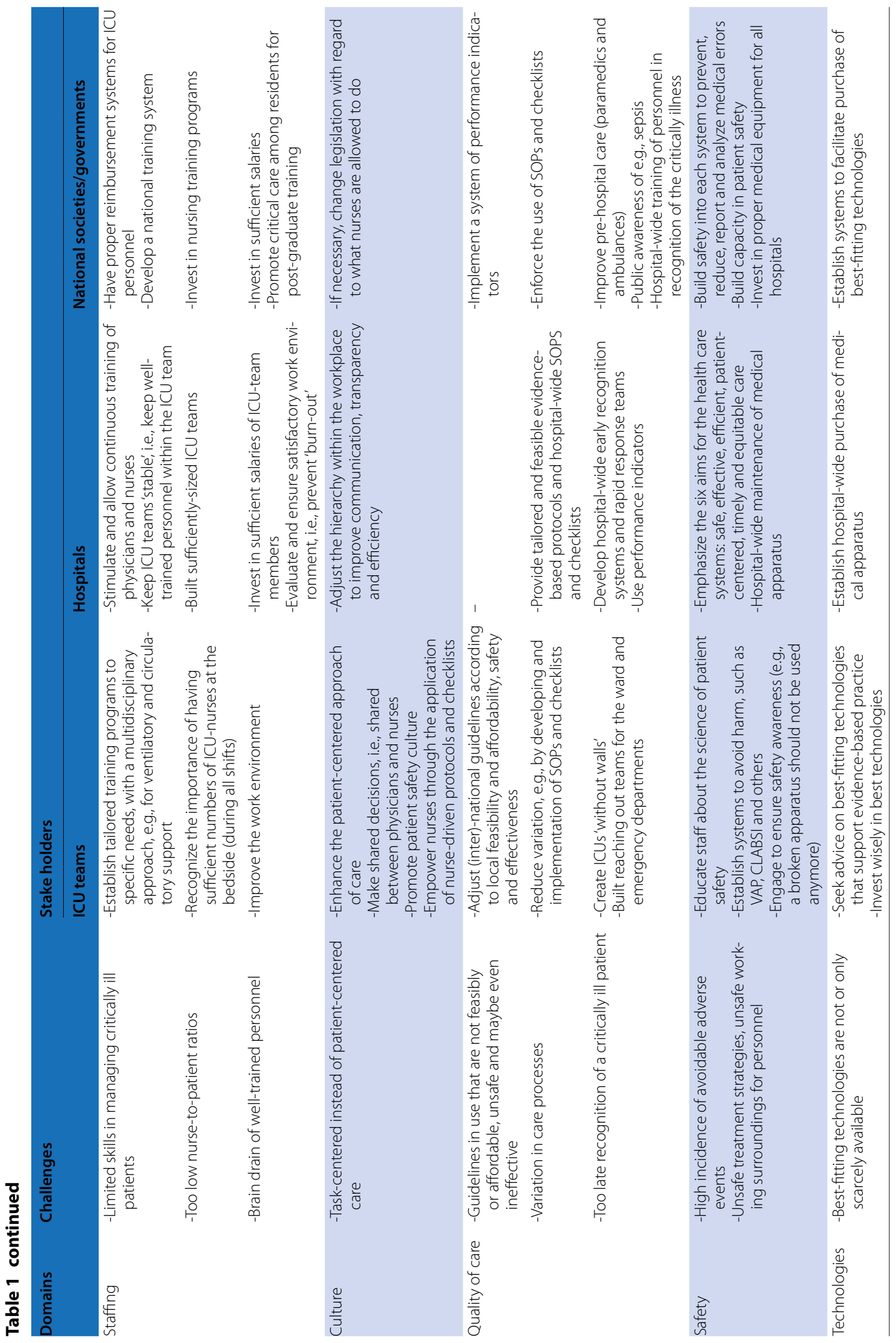




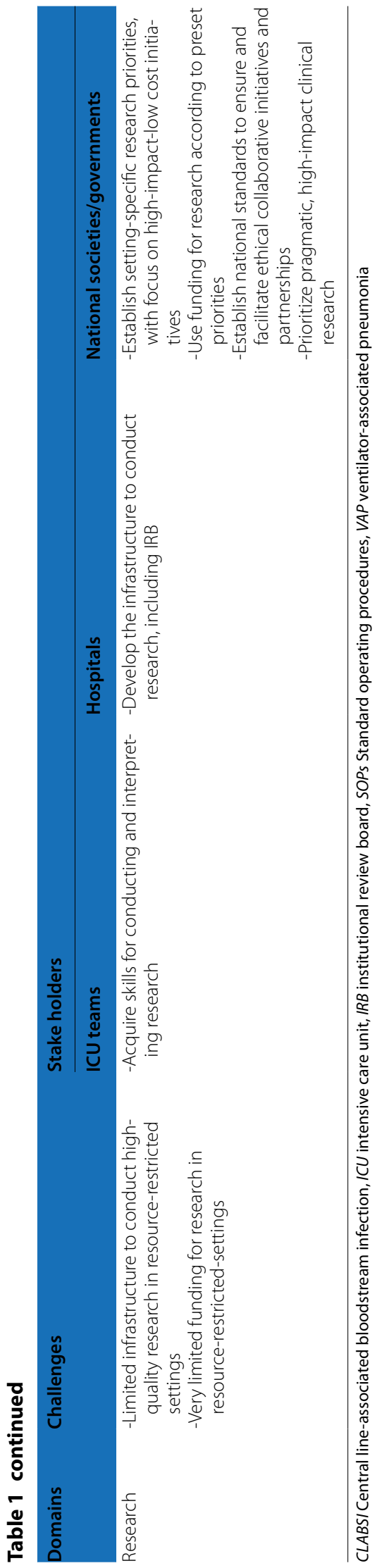

\section{Challenges in staffing}

In resource-restricted settings, critical care is not yet a well-developed specialty. Consequently, there is a great potential for incraasing the scope of training for physicians, nurses and other personnel. Training needs to focus on specific critical care skills, but it should also include basic management and organizational aspects, such as admission and discharge policies, multidisciplinary rounds, and proper documentation and handovers. Train-the-trainer approach will become increasingly important for sustainable training. In addition, resource-rich ICUs are increasingly committed to partner with resource-restricted ICUs for support and knowledge exchange. Several active examples include the initiatives by the World Health Organization, the Chinese University of Hong Kong, Médecins Sans Frontières, the National Intensive Care Surveillance (NICS) and our own academic groups.

\section{Quality improvements}

Wide variations in processes of care and outcomes exist among settings according to income level [7]. For example, mortality due to sepsis has decreased over the last decades to $20-40 \%$ in resource-rich settings, whereas mortality rates of up to $80 \%$ are still reported from resource-restricted regions [8]. This high mortality is attributed to late recognition of critical illness, poor translation of current knowledge [9] and poor ICU service structure. Low adherence to simple but effective evidencebased practices, such as low-tidal ventilation and the use of light sedation, is frequent in resource-restricted settings. A recent multicenter study demonstrated that implementing protocols in this setting is associated not only with lower mortality rates but also with an increased efficiency [10].

Clinical practice guidelines have been mainly based on evidence from resource-rich settings. Some of these recommendations, however, can be beyond the capability of resource-restricted ICUs and could even be dangerous, as is the case of aggressive fluid resuscitation (The FEAST trial) [11]. Treatment of infections should be setting-specific because resource-restricted countries have different pathogens, higher antibiotic resistance and less access to newer and expensive antimicrobials than resourcerich ones. An initiative from the Global Intensive Careworking group of the 'European Society of Intensive Care Medicine' has started to produce setting-specific recommendations [12], but this initiative is still hampered by absence of evidence for most recommendations used in resource-rich settings.

\section{Safety initiatives}

Avoidable adverse patient events are common in the ICUs [13]. The risk in resource-restricted settings is unknown but likely to be high. Therefore, a 
comprehensive approach for building safety into each system to prevent, reduce, report and analyze medical errors should be of great importance.

\section{Technological challenges}

Technology, such as telemedicine may play a significant role reducing the need for specialized staff [14]. Other measures, such as the use of remote application of checklists, are associated with improved adherence to best practices [15].

\section{Conclusion}

The journey toward excellent global critical care in 2050 is unlikely to be easy but is certainly going to be an exciting and interesting one. A comprehensive multifaceted approach is needed to improve the delivery of critical care globally, and such efforts are likely to translate to major improvement in patient outcome.

\section{Author details \\ ${ }^{1}$ Department of Intensive Care, King Saud bin Abdulaziz University for Health Sciences (KSAU-HS), PO Box 22490, Riyadh 11426, Saudi Arabia. ${ }^{2}$ King Abdul- lah International Medical Research Center (KAIMRC), Riyadh, Saudi Arabia. \\ ${ }^{3}$ Department of Intensive Care, Academic Medical Center, Amsterdam, The Netherlands. ${ }^{4}$ Laboratory of Experimental Intensive Care and Anesthesiology (LEICA), Academic Medical Center, Amsterdam, The Netherlands. ${ }^{5}$ Critical Care department, D'OR Institute for Research and Education, Rio De Janeiro, Brazil. ${ }^{6}$ Post-Graduate Program Federal University of Rio de Janeiro, Rio De Janeiro, Brazil.}

\section{Compliance with ethical standards}

\section{Conflicts of interest}

The authors report no conflict of interest.

Received: 2 November 2016 Accepted: 18 November 2016

Published online: 29 November 2016

\section{References}

1. United Nations. World population projected to reach 9.6 billion by 2050 .

13 June 2013. New York. http://www.un.org/en/development/desa/ news/population/un-report-world-population-projected-to-reach9-6-billion-by-2050.html. Accessed 27 Oct 2016
2. World Health Orgnization. Global Health Expenditure Database. http:// apps.who.int/nha/database/Key_Indicators/Index/en. Accessed 15 Nov 2016

3. Adhikari NK, Fowler RA, Bhagwanjee S, Rubenfeld GD (2010) Critical care and the global burden of critical illness in adults. Lancet 376(9749):1339-1346

4. Fowler RA, Fletcher T, Fischer WA 2nd et al (2014) Caring for critically il patients with ebola virus disease. Perspectives from West Africa. Am J Respir Crit Care Med 190(7):733-737

5. Firth P, Ttendo S (2012) Intensive care in low-income countries - a critical need. N Engl J Med 367(21):1974-1976

6. Austin S, Murthy S, Wunsch H et al (2014) Access to urban acute care services in high-vs. middle-income countries: an analysis of seven cities. Intensive Care Med 40(3):342-352

7. Arabi YM, Phua J, Koh Y et al (2016) Structure, organization, and delivery of critical care in Asian ICUs. Crit Care Med 44(10):e940-e948

8. Jacob ST, Moore CC, Banura P et al (2009) Severe sepsis in two Ugandan hospitals: a prospective observational study of management and outcomes in a predominantly HIV-1 infected population. PLoS One 4(11):e7782

9. Conde KA, Silva E, Silva CO et al (2013) Differences in sepsis treatment and outcomes between public and private hospitals in Brazil: a multicenter observational study. PLoS One 8(6):e64790

10. Soares M, Bozza FA, Angus DC et al (2015) Organizational characteristics, outcomes, and resource use in 78 Brazilian intensive care units: the ORCHESTRA study. Intensive Care Med 41(12):2149-2160

11. Maitland K, Kiguli S, Opoka RO et al (2011) Mortality after fluid bolus in African children with severe infection. N Engl J Med 364(26):2483-2495

12. Serpa Neto A, Schultz MJ, Festic E (2016) Ventilatory support of patients with sepsis or septic shock in resource-limited settings. Intensive Care Med 42(1):100-103

13. Arabi Y, Alamry A, Al Owais SM, Al-Dorzi H, Noushad S, Taher S (2012) Incident reporting at a tertiary care hospital in Saudi Arabia. J Patient Saf 8(2):87-681. doi:10.1097/PTS.0b013e31824badb7

14. Rincon TA, Bourke G, Seiver A (2011) Standardizing sepsis screening and management via a tele-ICU program improves patient care. Telemed J E Health 17(7):560-564

15. Kahn JM, Gunn SR, Lorenz HL, Alvarez J, Angus DC (2014) Impact of nurse-led remote screening and prompting for evidence-based practices in the ICU. Crit Care Med 42(4):896-904 